\title{
The Effect of Expressive Biosignals on Empathy and Closeness for a Stigmatized Group Member
}

\author{
FANNIE LIU, GEOFF KAUFMAN, and LAURA DABBISH, Carnegie Mellon University, USA
}

We explore the potential for expressive biosignals, or displays of sensed physiological data as social cues, to influence perceptions of a member of a stigmatized group. In a between-subjects experiment $(\mathrm{N}=62)$ participants read a fictional interview with a drug addict in prison, and rated their empathy and closeness with the interviewee. Participants were randomly assigned to read either 1) the transcript of the interview by itself, 2) the transcript with a text description of the interviewee's heart rate, or 3) the transcript with a graph of the interviewee's heart rate. Results demonstrate that providing information about heart rate can increase empathy in terms of emotional perspective-taking. Additionally, visualizing the heart rate as a graph, as opposed to text, can increase closeness. We discuss the implications of these results and present suggestions for future directions.

CCS Concepts: • Human-centered computing $\rightarrow$ Empirical studies in HCI; Empirical studies in collaborative and social computing.

Additional Key Words and Phrases: biosignals, emotion, empathy, interpersonal closeness, stigmatized groups

ACM Reference Format:

Fannie Liu, Geoff Kaufman, and Laura Dabbish. 2019. The Effect of Expressive Biosignals on Empathy and Closeness for a Stigmatized Group Member. Proc. ACM Hum.-Comput. Interact. 3, CSCW, Article 201 (November 2019), 17 pages. https://doi.org/10.1145/3359303

\section{INTRODUCTION}

We are living in an increasingly divided world, with political polarization perceived as a top global risk [28] and social schisms along dimensions of gender, race, social class, and numerous more on the rise. These growing divisions can diminish empathetic feelings towards members of particular groups [31], as evidenced by the increase in negativity towards people with different viewpoints [23] and the rise in the number of hate crimes over the last few years [25]. The decline in empathy may be exacerbated by the advent of technology-mediated communication [44], with some studies showing that technology use has a negative association with empathy [13]. For instance, sharing different views on social media can reduce empathy and deepen existing divisions [4]. It is crucial that we investigate new ways to design technologies to help us bridge, rather than widen, this divide and better understand each other. In our research, we pursue this goal by exploring the potential for new technologies that provide data on others' biosignals to promote empathy for and closeness with others.

The increasing ubiquity of consumer-grade wearable physiological sensors opens up the possibility for using personal sensed data, such as heart rate, to increase interpersonal understanding. Indeed, many wearable devices, such as smartwatches and fitness trackers, now come equipped

Authors' address: Fannie Liu, ffl@cs.cmu.edu; Geoff Kaufman, gfk@cs.cmu.edu; Laura Dabbish, dabbish@cs.cmu.edu, Carnegie Mellon University, Human-Computer Interaction, Pittsburgh, PA, 15213, USA.

Permission to make digital or hard copies of all or part of this work for personal or classroom use is granted without fee provided that copies are not made or distributed for profit or commercial advantage and that copies bear this notice and the full citation on the first page. Copyrights for components of this work owned by others than ACM must be honored. Abstracting with credit is permitted. To copy otherwise, or republish, to post on servers or to redistribute to lists, requires prior specific permission and/or a fee. Request permissions from permissions@acm.org.

(c) 2019 Association for Computing Machinery.

2573-0142/2019/11-ART201 \$15.00

https://doi.org/10.1145/3359303

Proc. ACM Hum.-Comput. Interact., Vol. 3, No. CSCW, Article 201. Publication date: November 2019. 
with functionality that allow for the displaying and sharing of biosignals in expressive and communicative ways. Because biosignals naturally change with fluctuations in cognitive and emotional states as a result of activity within the the autonomic nervous system, they have the potential to serve as powerful social cues to others' hearts and minds.

A small body of prior work has explored these expressive biosignals, beginning to show that information about another person's biosignals can help facilitate empathy and social awareness [35, $38,51]$ and, in some contexts, a greater sense of intimacy and connection [40,50]. These results point to potential opportunities for expressive biosignals to support interventions for bridging understanding with others, such in contexts in which feeling empathy or closeness with others is challenging (e.g., with members of socially distant groups, or physically remote others). However, the relationship between expressive biosignals and social connection with others is not wellunderstood. In particular, prior work often conflates the presence of biosignal information with their visual presentation $[35,38,40,50]$, where it is unclear what forms of biosignal information can affect empathy and closeness. Building on the foundation provided by expressive biosignals research, the present study aimed to investigate how both the presence of another individual's heart rate information and visualization of the heart rate data might affect empathy towards others and feelings of closeness between the self and other. Moreover, to provide a strong test of the impact of expressive biosignals on these outcomes, we explored their impact with a target other who belonged to a stigmatized group, for whom there are likely higher barriers to empathy and closeness to overcome. This work contributes an experiment that helps clarify the relationship between biosignals and social connection, specifically with a stigmatized group member, as well as the implications for these data to augment how people share experiences with each other online.

\section{RELATED WORK}

\subsection{Technological Interventions for Empathy}

To our knowledge, while interventions for empathy have been widely explored in social psychology literature (e.g., compassion meditation, skills workshops, role-playing) [73], research on technological interventions in this space is still limited. However, some research in HCI and CSCW has suggested the potential for features in existing technologies to affect aspects of empathy, as well as proposed novel interventions oriented around experiencing the perspective of another person.

Features of existing communication platforms have been shown to affect aspects of empathy for remote others. In video conferencing, Nguyen and Canny showed that displaying only the head, as opposed to the whole upper-body, can reduce empathy measured using self-report of cohesion, and speed of automatically helping a person in distress (i.e., when their pen drops) [57]. Thomas and Pemstein found webcam elevation influenced coordination and decisions made in one's own self-interest as opposed to benefiting a partner in a negotiation task [66]. Work on social media has shown that emotional and intimate self-disclosure have the potential to foster empathy and closeness between users, influencing social support [3,48]. For instance, Burke and colleagues found that hearing about a friend's troubles on Facebook causes friends to reply with more emotional and supportive comments [11]. However, these prior works have focused on different constructs related to empathy, such as cohesion, self-interest, and social support, rather than directly evaluating empathy.

Technology-based empathy interventions often take an experiential approach, allowing users to experience the world from the perspective of a distant other. Empathy glasses, for example, is a tool that allows you to view the world from the perspective of another person in real time [47]. In the same vein, virtual reality (VR), often described as the "ultimate empathy machine" [55], has immersive qualities that enable users to experience life from another person's perspective [9]. VR 
experiences have also shown potential to increase empathy for stigmatized groups. A Breathtaking Journey is an example of a mixed-reality game designed to foster empathy for refugees, suggesting empathy-arousing characteristics such as visceral engagement [45]. Becoming Homeless is a VR experience that has shown long-term effects on viewers' empathy towards homeless people in the form of improved attitudes and prosocial behavior [36]. VR is thus a promising platform on which to design empathy interventions. At the same time, VR interventions have some limitations. For instance, building games and stories require experts to design experiences around very specific narratives. Moreover, the experiences may be difficult to integrate into everyday expression and consumption of stories, which occurs on platforms like social media on a daily basis. In our work, we address these limitations by investigating a novel intervention, expressive biosignals, for stigmatized group members. In particular, we leverage the increasing ubiquity of wearable sensors and the ability to integrate biosignals data into existing technological platforms, such as social media.

\subsection{Expressive Biosignals and Empathy}

Prior literature suggests the potential for expressive biosignals to affect empathy for others. In particular, several qualitative studies that deploy expressive biosignal systems (both in the wild and in laboratory settings) have demonstrated that people tend to infer and be more aware of a close contact's emotional state through displays of heart rate and skin conductance [35, 38, 49, 51]

Few studies have quantitatively measured the potential for biosignals to enhance understanding of another person's emotional state. Merrill and Cheshire showed that graphs of elevated heart rate, as opposed to normal heart rate, can increase negative mood attribution (e.g., anxiety) to a partner in a trust game. These inferences can have behavioral consequences, including reduced cooperation. [54]. Their results demonstrate that different biosignal information can indeed affect perceptions of emotional state. However, it is still unclear how biosignals would affect mood attribution in comparison to not having biosignal information at all. Additionally, given the setup of the Prisoner Dilemma game, which provides a context for trust and cooperation, the study does not directly investigate whether biosignals impact empathy.

To our knowledge, only one recent study quantitatively investigates the relationship between expressive biosignals and empathy. In a laboratory experiment, Curran and colleagues explored the influence of biosignal information on empathic accuracy and "state empathy." Participants watched a VR narrative from a target person's field of view with or without a graph of the target's skin conductance. Empathic accuracy was measured based on how much participants' ratings of the target's feelings (based on their valence, e.g., "very positive") matched the target's own ratings of their feelings, while state empathy was measured according to how much participants felt they were in the shoes of the target. Surprisingly, they found that biosignal information actually decreased empathic accuracy and did not affect state empathy [19]. There are several possible explanations for this result, including that the VR film may not provide sufficient context to infer emotional state, or by itself may result in a high baseline level of empathy. In addition, empathic accuracy and state empathy as measures are limited in their ability to capture empathy towards another person, where the former focuses on alignment in the intensity of valence, and the latter focuses on cognitively simulating a person's situation.

In our research, we choose a different operationalization of empathy than empathic accuracy and state empathy. Empathy as conceptualized in the literature includes emotional perspective-taking, or the inference of another person's emotional state in a given context [24, 65], and empathic concern, or feelings of sympathy or compassion towards another person [6]. Biosignals may still influence emotional perspective-taking and empathic concern for another person. Indeed, prior work suggests that people primarily infer emotional states from biosignals [54], and that biosignals 
can promote sympathy with and desire to support others [51]. In the current work, we sought to directly examine the relationship between biosignals and these two components of empathy.

\subsection{Expressive Biosignals and Interpersonal Closeness}

Research has also shown that expressive biosignals can affect closeness with others. For instance, systems such as rings or smartwatches where you can feel or see a partner's heart rate can improve feelings of being connected with remote friends and family $[49,51,56,63,69]$. Janssen and colleagues demonstrate that biosignals can also increase feelings of closeness with strangers. They investigated how the sound of an unknown partner's heartbeat affects intimacy compared to silence. They found that it can have comparable levels of intimacy as interpersonal distance and mutual gaze, which are known to be highly intimate [40].

At the same time, different ways of conveying biosignals information could have differing effects on closeness. In a mixed-methods experiment involving six brain activity visualizations, Liu and colleagues show that different visualizations can lead to different feelings of connectedness with a stranger [49]. Moreover, in a study where participants sent texts of their heart rate to others, Liu and colleagues found that participants desired more vivid and expressive visualizations for their heart rate than simple text denoting their beats per minute [50]. Animated depictions of heart rate on a smartwatch have also shown the potential to increase the salience of another person [51].

Results from these prior works suggest a need to be selective about how to convey biosignals information, especially when considering the relationship or context of communication between interactants. For instance, animated visualizations of biosignals could be useful for bringing people closer or making them more aware of each other's presence, while text descriptions of heart rate may be more appropriate for situations in which intimacy is not desired (e.g., strictly professional relationships). In the present work, we explore the potential for visualizing biosignals, as opposed to simply describing in text, on interpersonal closeness and salience of the partner.

\section{RESEARCH CONTEXT}

\subsection{Stigma and Drug Addiction}

To understand the effects of expressive biosignals on empathy and closeness, we explore this relationship in the context of perceptions of stigmatized group members. Though research has defined stigma in various ways, in our work we refer to a stigmatized person as one who is "socially discredited" [33] or has "undesirable characteristics" that "set [them] apart from others" [42]. According to Goffman, stigmatized people can include those with "a known record of mental disorder, imprisonment, addiction, alcoholism, homosexuality, unemployment, suicidal attempts, and radical political behavior" [33].

For the purposes of our study, we focus on empathy and closeness towards a convicted drug addict. In line with Goffman's claim, research has shown evidence for the stigmatization of drug addicts by the general public, where people with addiction are more likely to be viewed as dangerous and be blamed for their condition [16, 52, 72]. A United States national survey with 709 participants found that the public had significantly more negative views on drug addiction as compared to mental illness, where respondants were significantly less willing to work with a drug addict, more likely to accept discrimination against drug addicts (e.g., denial of employment and housing), and more likely to oppose public policies supporting drug addicts (e.g., government spending for treatment), as compared to people with mental illness [5]. Stigma towards drug addicts is a major issue that can affect their ability to access treatment, discouraging addicts to seek treatment and affecting health professionals' willingness to provide treatment [17, 67]. 


\subsection{Reduced Empathy towards Stigmatized Groups}

The challenge of building empathy toward members of stigmatized groups is a firmly established area of inquiry within social psychology. Despite its benefits for facilitating interpersonal and intergroup harmony and mutual understanding, empathy is neither a universal nor an automatic response. Social boundaries created by in-group/out-group distinctions on social identity dimensions (such as race, religion, or political affiliation) as well as differences in background or experience have been shown to diminish behavioral, neural, and physiological expressions of empathy for the other $[14,15,29,73]$. These failures of empathy, when an individual could potentially experience empathy toward the other but does not because of salient social and psychological factors, are particularly difficult to bridge when the target other is a member of a stigmatized population. For example, Decety and colleagues found that participants who watched brief video clips of individuals experiencing physical pain attributed lower levels of pain to them if they had been told beforehand that they were AIDS patients than if they were not given this information [21]. Of particular relevance to the present research is that research on empathy and stigma has often positioned particular stigmatized populations, including drug addicts, as "extreme out-groups" [26] that typically elicit patterns of dehumanization, including reduced levels of compassion and recognition of group members' mental states [12, 27].

\subsection{Research Inspiration}

The present study is a conceptual replication of one initially conduced by psychologist Daniel Batson and colleagues [7]. In their work, participants were asked to listen to an interview conducted with a drug addict and were provided with explicit instructions either to try to remain objective or to imagine the interviewee's feelings while listening to the account. This study found that those participants who had been instructed to imagine the interviewee's feelings showed higher rates of empathy and prosocial behavior (donating to a counseling service for drug addicts) as well as more positive attitudes toward drug addicts as a whole.

We chose Batson and colleagues' work as a basis for our research for several reasons. First, experimental control is necessary to isolate the effects of biosignals on empathy and closeness. External factors in-the-wild or aspects of synchronous communication channels (e.g., immediacy or appearance in face-to-face, text, and video) are more likely to confound results. Moreover, we wanted to draw from existing experimental research known to observe a clear increase in empathy, such that we could investigate whether biosignals could have the same effect. At the same time, using a vignette setup increases the ecological validity of the study. Textual vignettes are a common form in which people share and consume stories today, including on social media, blogs, discussion forums, and other online platforms. For example, similar to how Batson and colleagues used an interview with a drug addict, Humans of New York [64], a highly popular photo blog, shares stories (often about personal struggles) through text excerpts of street interviews conducted by the blogger, Brandon Stanton. Prior HCI research has also used vignettes in a similar way: Andalibi and Forte, for instance, used vignettes in the form of social media posts as a prompt to understand how people respond to sensitive disclosures online [1].

Representative of the typical approach to inducing empathy or perspective-taking, Batson and colleagues' study provides an explicit instruction to attempt to empathize with a target other. Similarly, research on perspective-taking, or achieving an understanding of another's mindset or experiences, has utilized explicit instructions to achieve similar outcomes for out-groups (e.g., imagining a day in the life of members of other races or nationalities) [20, 32, 70]. Finally, a third line of work has focused on strategically crafting personal narratives describing the experiences of stigmatized others in ways that induce greater connections and empathy, including adding cues to 
similarity between self and other early in the narrative or delaying the disclosure of the other's stigmatized identity to allow a connection to form prior to the revelation [43]. In the present work, we investigate whether simply providing access to a stigmatized other's heart rate data could serve a similar function to these experimental inductions: to provide a cue to attempt to connect with and to understand the perspective and emotional experience of the other.

\subsection{Hypotheses}

3.4.1 Presence of Biosignals and Empathy. We expect biosignals to positively influence empathy towards stigmatized others. In light of prior work on biosignals, we hypothesize that biosignals increase empathy, as defined by emotional perspective-taking and empathic concern.

H1. The presence of biosignal information will increase empathy.

Our work also explores how expressive biosignals affect behaviors and perceptions known to be related to increased empathy. Drawing from Batson and colleagues' original study [7], we predict that the presence of biosignal information will promote prosocial behavior and improved attitudes towards stigmatized groups.

$\mathrm{H} 2$. The presence of biosignal information will increase prosocial behavior.

H3. The presence of biosignal information will improve attitudes towards stigmatized groups.

3.4.2 Visualizing Biosignals and Closeness. Prior work suggests that text depictions of biosignals may not be as vivid as an animated visualization or audio representation [50]. Thus, we expect that visualizing biosignals as a graph, as opposed to describing the biosignals in text, will increase closeness with and salience of a member of a stigmatized group. We choose a graph to visualize biosignals for two reasons. First, graphs are commonly used to visualize biosignals in both research $[18,54,61]$ and consumer applications that track biosignals, such as Fitbit and Muse. Therefore, the graph provides a suitable baseline case that would be familiar to participants as well as ecologically valid with respect to how biosignals are typically visualized in the real-world today. Second, a graph would help control the information conveyed to participants. Prior work shows that design characteristics of different biosignals visualizations can have a significant effect on interpersonal perceptions [49]. For instance, perceptions could be confounded by prior associations people have with representations like an emoji or audio or vibrations of a heartbeat, which are all culturally embedded. In our work, using a graph to visualize biosignal information, we hypothesize:

H4. Visualizing biosignal information will increase interpersonal closeness more than presenting biosignals information as text.

H5. Visualizing biosignal information will increase the salience of a target more than presenting biosignal information as text.

\section{METHODS}

We ran a between-subjects experiment where participants read a series of interview excerpts. Participants were randomly assigned to one of three conditions corresponding to the type of heart rate data that accompanied the excerpts: 1) No HR, meaning no biosignal information was provided at all, 2) HR Caption, meaning a caption about the interviewee's heart rate was provided, and 3) $H R$ Graph, meaning a caption about the interviewee's heart rate and a heart rate graph were provided.

\subsection{Participants}

We recruited 72 participants from Amazon Mechanical Turk. We removed 10 participants who did not read or understand the materials in the online survey, based on their responses to the attention check questions we included. The remaining 62 participants included 24 females and 38 males, with 
an age range of 22 to 69 years old $\left(M_{\text {age }}=37.47, S D_{\text {age }}=10.16\right)$. All participants were US Citizens. There were 23 participants in the No HR condition, 18 in the HR Caption condition, and 21 in the $H R$ Graph condition. Participants were compensated $\$ 3.00$ for participating in the study.

\subsection{Procedure}

We adapted our procedure from Batson and colleagues' prior work on empathy towards stigmatized groups, specifically drug addicts [7]. To increase ecological validity and believability, we modernized their materials with the cover story that we are creating an online platform for people to share personal experience with new forms of data (in this case, physiological data). The platform would be similar to existing online story-sharing websites such as forums and social media. Participants were told that we previously interviewed and recorded data from people from a variety of backgrounds, and that the purpose for the study was to collect reactions to the stories of our prior interviewees.

Participants completed the study by taking a 15-minute online survey. In the first part of the survey, participants read a transcript of a fictional interview. The fictional interview was conducted with a 22-year old man named Jared who was serving a 7-year sentence for possession and sale of heroin. In the transcript, Jared describes how he was introduced to heroin and became addicted, and his desire to start a better life after finishing his prison sentence. Though Batson and colleagues' original study used audio for Jared's interview, we decided to use text instead of richer channels, like audio or video, in order to reduce extra information and signals available to participants that could confound the results (e.g., Jared's voice or appearance).

In order to manipulate the presence of biosignal information we included two biosignal information conditions, one with (HR Graph) and one without a visual (HR Caption). Heart rate information is known to be ambiguous and can be interpreted in various ways [53]. In fact, in our preliminary pilots, many participants cited withdrawal as a cause for the changes in Jared's heart rate. To guide participants' interpretations around Jared's feelings rather than drugs, participants were told that Jared shared about his experience and feelings as part of the interview. This was done in all three conditions to ensure that participants in each condition had the same background information for the interviews.

In the HR Graph condition, participants viewed an animated graph of Jared's heart rate (represented by a per-beat graph). To ensure that participants in both conditions received the same amount of heart rate information, the provided caption was the same (that Jared's heart rate was elevated at an average beats per minute significantly above his baseline) and the graph was kept as neutral as possible (e.g., it kept a static rate of change and did not contain drastic peaks). To reduce potential differences in how participants related the graph to different sections of the interview, we split the transcript into four excerpts, where each excerpt included a question asked by the interviewer and Jared's response to that question. Each excerpt was shown with a section of the graph. To reduce variation between the graph information seen with each excerpt, graph sections changed at a similar rate and had a similar number of peaks. This was done in order to control the information we presented, such that Jared's heart rate would appear consistently high throughout the interview. An example screenshot of an excerpt in the HR Graph condition can be seen in Figure 1. Additionally, to control for the time spent reading Jared's responses and viewing the graph, participants were given 20 seconds for each excerpt. The HR Caption and No HR conditions followed a similar structure as the HR Graph condition, except without the graph and without the graph and caption, respectively.

After reading the transcript, participants answered several questions about the excerpts and their demographic information. The end of the survey included a debrief, explaining the purpose of the study and that Jared is a fictional person. 


\begin{abstract}
Please read the interview excerpt below carefully. After 20 seconds you will be able to press next.

Below: Jared's heart rate during his response to the question below. During this portion of the interview, Jared's heart rate was elevated, averaging at $87 \mathrm{bpm}$, which was significantly above his baseline.

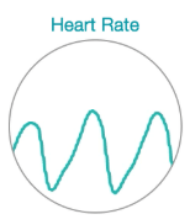

Interviewer (l): How did you first get introduced to heroin?

Jared (J): Well, in my junior year in high school, I started hanging out with some guys after school. I knew that they weren't the best crowd, but they weren't really bad. Sometimes we'd drink a few beers - sometimes more than a few-and sometimes smoke some pot, but nothing major. (pause) My parents, they didn't care; they were too busy fighting each other and working on the divorce-they're split now. (pause) Then one night Erik, an older guy -25 maybe - he was there and had a bag of heroin and asked if anyone would like try it out. After a couple of other guys tried it, I figured I would too. So, that's when I took my first hit. I was surprised; it felt really good. Of course, it wasn't like I was addicted or anything after shooting up just once.
\end{abstract}

Fig. 1. Example screenshot of the interview excerpt in the HR Graph condition.

\title{
4.3 Measures
}

Participants answered questions related to their experience reading the excerpts and, if they were in either the HR Caption or HR Graph conditions, viewing Jared's heart rate information. They were also asked to answer questions about their perceptions of Jared and more generally, drug addicts. We included the following measures:

Empathy. For our purposes, we operationalized empathy in two ways: empathic concern and emotional perspective-taking. Empathic concern refers to feeling for another person in need, and consists of emotions such as sympathy, compassion, warmth, tenderness, softheartedness, and being moved [6]. We included the six empathic concern items as part of a 15-item question asking participants to rate their feelings towards Jared. Emotional perspective-taking refers to inferring another person's emotional state based on their context [65]. To measure emotional perspectivetaking, we used a shortened version of the PANAS scale, a widely used measure for perceptions of emotions, with items for positive and negative emotions [68]. We included five positive (excited, strong, enthusiastic, proud, determined) and five negative emotions (distressed, scared, hostile, ashamed, nervous) to cover a diverse and relevant set of emotions to Jared's story.

Prosocial Behavior. Batson and colleague's prior work using the Jared interview suggests a positive relationship between empathy towards a stigmatized group member and prosocial behavior towards the whole group [7]. We included two measures of prosocial behavior: one that aligned with Batson and colleagues' use of donations as a measure of prosocial behavior, where MTurk participants could donate a portion of their HIT reward (ranging from $\$ 0$ to $\$ 2.50$ in increments of $\$ 0.50$ ) to a 
local Addiction Counseling Service (with no connections to Jared), and another where they could leave a message of support for Jared.

Attitudes towards Stigmatized Groups. Batson and colleagues' prior work also suggests a positive relationship between empathy towards a stigmatized group member and attitudes towards the whole group [7, 8]. Thus, we included a scale to measure attitudes towards people addicted to hard drugs (e.g., "People addicted to hard drugs lack self-control and inner strength.", "People who end up addicted to hard drugs only have themselves to blame" [7, 10]).

Closeness. To measure interpersonal closeness, we used the Inclusion of the Other in the Self (IOS) scale [2], asking participants to use the scale to rate how they saw themselves in relation to Jared, and how similar to Jared they felt.

Salience of Fared. To measure the salience of Jared, we included scales for social presence and experience taking. Social presence is the "degree of salience" of another person [62]. We included scales that highlight several aspects of social presence, including how how "real" (as opposed to abstract) Jared seemed [46], the participants' ability to assess the Jared's reactions and get to know Jared [58], and the immediacy and intimacy of the excerpts [34]. Experience taking refers to the extent to which someone simulates a character in a narrative. This seven question scale includes items such as "I found myself feeling what Jared was feeling." [43].

Experience with Hard Drugs. Participants' perceptions of Jared could be affected by their knowledge of and experience with hard drugs or users of hard drugs. Therefore, we included one question to measure participants' experience with hard drugs, asking participants to optionally respond to whether they or their loved ones (e.g., friends or family) had any experience with hard drugs.

Attention Check. Finally, we included several questions to ensure that participants were reading and understanding the interview and graph. These included questions about details that Jared described in the interview itself (e.g., "What is Jared planning to do after his prison sentence?"), and details about the graph (e.g., "What was the graph plotting?").

\section{RESULTS}

For each of the outcome measures reported we employed a one-way analysis of covariance (ANCOVA) and two planned contrasts to evaluate our hypotheses related to the presence and visualization of heart rate data: 1) a presence contrast that compared the No HR condition to the average of the HR Caption and HR Graph conditions (reflected in contrast coefficients of -1, .5, and .5, respectively), and 2) a visualization contrast that compared the HR Caption and HR Graph conditions (with contrast coefficients of -1 and 1). We also applied Bonferonni correction for ANOVAs on individual items within scales (i.e., dividing the critical p-value by the number of comparisons made). To control for effects of experience with hard drugs, we included responses to the experience with hard drugs measure as a covariate.

\subsection{Empathy}

We conducted a one-way ANCOVA on the empathic concern and PANAS scales. We analyzed the PANAS scale by separating the sets of positive and negative emotions. Additionally, since Jared's story dealt with specific emotions, we analyzed individual items in the PANAS scale to investigate the most relevant emotions to the narrative. Results from the ANCOVA test on the empathic concern scale and PANAS positive or negative affect scales did not indicate significant differences between the conditions at $p \leq 0.05$. However, results from the ANCOVA test on the individual items of the PANAS indicated significant differences between conditions on two emotions (at $p \leq 0.01$ ): 
Table 1. Means of measures with significant differences across conditions at $p \leq 0.05$ (with Bonferonni correction for individual scale items). Empathy (PANAS) measures compared No HR vs HR Caption and HR Graph, while Closeness measures compared HR Caption vs HR Graph.

\begin{tabular}{llll}
\hline Measure & No HR & HR Caption & HR Graph \\
\hline PANAS - JARED'S FEELINGS (/5) & & & \\
Nervous & $2.30(1.02)$ & $3.39(1.04)$ & $3.29(1.06)$ \\
Strong & $2.57(1.08)$ & $1.83(0.92)$ & $1.62(0.74)$ \\
\hline IOS - CLOSENESS TO JARED (/7) & & & \\
Closeness in Relation & $2.17(1.07)$ & $2.00(1.03)$ & $3.19(1.72)$ \\
Similarity & $2.09(1.28)$ & $1.67(1.03)$ & $2.71(1.52)$ \\
\hline
\end{tabular}

nervousness $(F(2,58)=7.17, p=0.002)$ and strength $(F(2,58)=6.12, p=0.004)$. Results from the planned presence contrast indicated significant increase in participants' perceptions of Jared's nervousness $(F(1,58)=14.34, p<0.001)$ and a significant decrease in perceptions of strength $(F(1,58)=11.57, p=0.001)$ in the two $H R$ conditions compared to the No HR condition. These results partially support $\mathrm{H} 1$, where providing information on biosignals affected empathy in terms of emotional perspective-taking, but not empathic concern.

\subsection{Prosocial Behavior and Attitudes}

We conducted a one-way ANCOVA on donation amount and attitudes towards people addicted to hard drugs, and chi-square tests on whether participants donated at all or left a message of support. Results from these tests did not indicate a significant difference in participants' prosocial behavior (both for leaving donations and support messages) or attitudes towards people addicted to hard drugs between conditions. We reject $\mathrm{H} 2$ and $\mathrm{H} 3$, which predicted that the presence of biosignals information will increase prosocial behavior and improve attitudes towards stigmatized groups.

\subsection{Interpersonal Closeness}

We conducted a one-way ANOVA on the IOS closeness scale ${ }^{1}$. We also ran the visualization contrast on both scales. Results from the ANOVA test indicated a significant difference between conditions for how close participants felt in relation to Jared $(F(2,59)=4.86, p=0.01)$. Similarly, results from the ANCOVA test indicated a significant difference between conditions for how similar participants felt to Jared $(F(2,58)=3.26, p=0.05)$. Results from the planned contrast indicated that participants felt significantly closer in relation to Jared $\left(t\left(33.34^{2}\right)=2.66, p=0.01\right)$ and more similar to Jared $(F(1,58)=6.35, p=0.01)$ in the HR Graph condition compared to the HR Caption condition. These results support $\mathrm{H} 4$, which predicted that visualizing biosignals, as opposed to presenting biosignals as text, will increase interpersonal closeness.

\subsection{Salience of Jared}

We conducted a one-way ANCOVA for each of the three social presence scales and the experience taking scales, including on both individual items from the scales. None of these measures indicated significant differences between the conditions at $p \leq 0.05$. Thus, we reject $\mathrm{H} 5$, where visualizing biosignals information did not increase the salience of Jared more than simply presenting the information as text.

\footnotetext{
${ }^{1}$ Since this measure did not meet the assumption of equal variances at $p=0.007$, we ran an ANOVA instead of an ANCOVA, and a one-way ANCOVA on the IOS similarity scale

${ }^{2}$ We note that this result has a different degree of freedom because it does not assume equal variances.
} 


\section{DISCUSSION}

Altogether, our results support and extend prior work by demonstrating the potential for expressive biosignals to function as a novel intervention for empathy and closeness. In particular, we show that providing information about heart rate can increase emotional perspective-taking for a stigmatized group member. Additionally, visualizing heart rate, as opposed to just describing it in text, can promote interpersonal closeness with a stigmatized group member.

\subsection{Effects of Heart Rate Information on Empathy}

Our results partially supported H1, which predicted that heart rate information, presented as a caption or in a graph, will increase empathy. We measured empathy in two ways: emotional perspective-taking and empathic concern. Participants reported significantly higher emotional perspective-taking when they saw information about Jared's heart rate alongside the interview, where Jared's nervousness and lack of strength was more salient. This supports and provides quantitative evidence for prior work [35, 38, 50,51], showing that the presence of biosignal information provides cues that people use to infer someone else's emotional state based on context. Additionally, we extend prior work by highlighting the potential for biosignals to affect empathy for a stigmatized group member.

However, our results did not show significant effects on empathic concern. One possible explanation is the limited modality of the interview, which was shown in text. Unlike Batson and colleagues' original study [7], we purposefully did not use an audio recording of Jared's whole interview. We included only text excerpts from the interview. Given that audio is more contextually rich than text [22], potentially an audio recording combined with heart rate data is necessary to observe greater effects. Future work should explore comparing different modalities of communication (e.g., text, audio, video) with the presence or absence of heart rate data. Additionally, our results on emotional perspective-taking showed significant effects only for perceptions of Jared's nervousness and strength. Potentially, the salience of these emotions may not be as relevant to empathic concern as others, such as regret or worry (which are emotions present in narratives used in prior empathy work [8]). In our future work, we plan to explore narratives that provide context for inferring different emotional states from heart rate. Another alternative explanation is the cultural context of the participants. Though we had asked participants about their experience with hard drugs or users of hard drugs, participants' attitudes towards drug addicts could have been indirectly affected by their cultural understanding of drug addiction (e.g., varying knowledge of drug addiction per US region, the recent declaration of the opioid crisis as a national emergency). Future work should explore the effect of biosignals information on perceptions of drug addicts in different cultures and regions, as well as perceptions of other stigmatized groups.

We did not observe an influence of biosignal presence on prosocial behavior or attitudes towards stigmatized groups. Given that we did not observe significant effects on empathic concern, this aligns with prior work about empathic concern and its related effects [7,8]. Additionally, our analysis yielded a mean of $\$ 0.22$ in donations, $7 \%$ of the amount they could donate (their payment for the task), as compared to $13 \%$ in the low empathy condition in Batson's original study [7], or roughly $10 \%$ in some studies on charitable giving $[59,60]$. This is potentially due to limitations in our donation measure. The original study asked student participants if they were interested in allocating some of a (fictional) university committee's budget to an Addiction Counseling Service. In contrast, we asked MTurk participants to donate some of their personal funds from completing the task. Since participants were incentivized to participate based on money compensation, they were likely more attuned to their own funds than students were to their school committee's funds. We 
plan to conduct follow-up studies to understand effects on different measures of helping, drawing from a university population rather than MTurk.

\subsection{Effects of Heart Rate Visualization on Closeness}

We show that a heart rate visualization, as opposed to just text describing a heart rate, can promote interpersonal closeness with a stigmatized group member: IOS ratings for participants' relation to Jared and similarity to Jared were significantly higher for the HR Graph condition than the HR Caption condition. This suggests that while simply providing heart rate information in different forms can help increase awareness of someone else's state, visualizing the heart rate changes is important for improving the relationship with that person. Moreover, our results extend past work showing the effects of audio heart rate cues on closeness [40]; we demonstrate that visual heart rate cues can similarly affect closeness, including for socially distant others.

At the same time, the heart rate visualization did not have significant effects on participants' ratings for social presence or experience taking. Potentially, the visualization we chose for heart rate, a graph, did not provide a vivid representation of Jared's feelings. Indeed, the form of a screen-based graph of raw data may be too "clinical," as compared to more physical or abstract forms such as a colored light bulb [49] or worn watch with haptic feedback [51]. Future work should explore which visualization schemes for heart rate best provide cues that could increase the overall salience of the other person.

\subsection{Implications for Technology}

Our results support that expressive biosignals can influence perceptions of stigmatized others whose stories they read online. Online platforms are increasingly used to share and consume personal stories. These include popular social media sites like Twitter that encourage broad discourse using hashtags, blogs that collect and disseminate stories like Humans of New York or Hollaback!, anonymous forums like microaggressions.com, or Facebook groups that function as online support for health-related issues. While these platforms provide opportunities and outlets for people to share their experiences, the audience who will read or respond to those stories may not necessarily be able to connect with or understand them-as evidenced by known challenges in polarization, harassment, implicit biases online [4, 30, 41]. This can be exacerbated with the growing possibility to encounter diverse others online who may perceive them as stigmatized or part of an out-group.

The present research on expressive biosignals introduces a promising new direction for the use of physiological data to mitigate this divide on online platforms. Our results show that biosignals like heart rate can help people understand others' emotional state, which otherwise can be difficult to recognize when communicating at a distance. The presence of biosignals could augment stories shared online by increasing their emotional quality and helping people better express themselves. This could be especially helpful for highly emotional experiences that may be unfamiliar to others or difficult for someone to convey through just words. For instance, one might share an experience of stereotype threat alongside their heart rate information during the experience. The heart rate information could help others who are not familiar with stereotype threat to better recognize and understand its emotional effects. Additionally, our results suggest that incorporating vivid visualization schemes for biosignals would heighten feelings of closeness that may be difficult to experience with someone in a different social group. Platforms that incorporate biosignal data into story-sharing should consider representations beyond raw numbers, such as dynamic graphs, which could help readers of stories better connect with the storyteller. Designers of existing and future online platforms for story-sharing should explore the potential for integrating biosignals as a novel cue to enhance the creation and consumption of personal stories, as well as connection with storytellers. 
With this call to action, however, we offer two crucial caveats. First, any application that entails the collection and display of personal data should prioritize the agency of users to preserve their own privacy and to choose how and with whom their biosignals are shared. People may have concerns about the intrusiveness of this intimate and sensitive data $[49,50]$; thus, we need to understand the privacy controls that are necessary for this type of data [71]. In the present work, for example, we explicitly state that Jared agreed to share his heart rate data with participants. Second, because of the inherent ambiguity of biosignals and the potential authority imbued to systems that collect and display them, designers should provide users with the guidance for critical reflection and discussion of biosignals as part of users' interpretive process [37, 39].

\section{LIMITATIONS AND FUTURE WORK}

The present work revealed that accompanying a personal narrative from a member of a stigmatized group (individuals battling drug addiction) with access to that person's heart rate data and visualizations of that data facilitated greater emotional perspective-taking and perceived closeness, respectively. At the same time, the results did not show an impact of heart rate data on several key outcomes, most notably empathic concern and prosocial behavior. In future work, we aim to replicate the findings from the present study to other stigmatized groups (e.g., racial out-groups or members of different political parties) and, moreover, compare the relative impact of heart rate information and visualizations on perceptions and responses to non-stigmatized groups, as compared to stigmatized groups (or more broadly, between in-group and out-group members).

Additionally, we aim to extend the present findings on emotional perspective-taking and closeness through iterations on our study setup. This includes investigating other emotional states (beyond those studied in the present work), such as personal narratives about the uncertainty and anxiety faced by particular stigmatized out-groups (e.g., racial minorities) who experience prejudice and discrimination. Moreover, we plan to explore the design of different visualization schemes that might be more conducive to increasing connection with and salience of another person, including embodied, physical, or sensory representations of biosignals $[40,49,51]$ that have shown promise in prior work.

Finally, we reiterate that in the present research we prioritized high experimental control and internal validity, by relying on a previously established study protocol as a foundation and attempting to isolate the impact of biosignals displays on empathy and closeness. Future work will aim to establish the external validity of these findings via field studies conducted in more naturalistic settings. To this end, part of our follow-up work involves deploying and evaluating an online platform designed to collect personal stories from college students to be shared with their peers, in which we will continue to investigate the effects of biosignal visualizations on empathy and prosocial behavior with the aim of creating a forum that students will actually use to share their experiences and to provide and receive social support.

\section{CONCLUSION}

We contribute one of the first experiments investigating the effects of expressive biosignals on empathy for and closeness with a stigmatized group member. We find that providing information about heart rate can increase empathy in terms of emotional perspective-taking, and interpersonal closeness can increase with a visualization of that information. Given the increasing number of opportunities for individuals to share their experiences online (e.g., on anonymous forums like microaggressions.com) and to both encounter and interact with members of diverse groups, our results have implications for helping viewers of these stories better understand them and connect with the people behind them through biosignals. Our future work will further explore this potential, including through new research directions around platforms for shared personal experiences and 
different expressive biosignals visualization schemes that elicit greater feelings of empathy and closeness with others.

\section{ACKNOWLEDGMENTS}

We would like to thank Dr. C. Daniel Batson for sending us the interview transcript used in his original vignette study. This work is supported by the National Science Foundation under CRI-1205539.

\section{REFERENCES}

[1] Nazanin Andalibi and Andrea Forte. 2018. Responding to Sensitive Disclosures on Social Media: A Decision-Making Framework. ACM Transactions on Computer-Human Interaction (TOCHI) 25, 6 (2018), 31. https://doi.org/10.1145/3241044

[2] Arthur Aron, Elaine N Aron, and Danny Smollan. 1992. Inclusion of Other in the Self Scale and the structure of interpersonal closeness. Journal of Personality and Social Psychology 63, 4 (1992), 596. https://doi.org/10.1037/0022-3514. 63.4 .596

[3] Sangwon Bae, Jinkyu Jang, and Jinwoo Kim. 2013. Good Samaritans on social network services: Effects of shared context information on social supports for strangers. International fournal of Human-Computer Studies 71, 9 (2013), 900-918. https://doi.org/10.1016/j.ijhcs.2013.04.004

[4] Christopher A. Bail, Lisa P. Argyle, Taylor W. Brown, John P. Bumpus, Haohan Chen, M. B. Fallin Hunzaker, Jaemin Lee, Marcus Mann, Friedolin Merhout, and Alexander Volfovsky. 2018. Exposure to opposing views on social media can increase political polarization. Proceedings of the National Academy of Science 115, 37 (2018), 9216-9221. https: //doi.org/10.1073/pnas.1804840115

[5] Colleen L Barry, Emma E McGinty, Bernice A Pescosolido, and Howard H Goldman. 2014. Stigma, Discrimination, Treatment Effectiveness and Policy Support: Comparing Public Views about Drug Addiction with Mental Illness. Psychiatric Services 65, 10 (2014), 1269-1272. https://doi.org/10.1176/appi.ps.201400140

[6] C Daniel Batson and Nadia Y Ahmad. 2009. Using Empathy to Improve Intergroup Attitudes and Relations. Social Issues and Policy Review 3, 1 (2009), 141-177. https://doi.org/10.1111/j.1751-2409.2009.01013.x

[7] C Daniel Batson, Johee Chang, Ryan Orr, and Jennifer Rowland. 2002. Empathy, Attitudes, and Action: Can Feeling for a Member of a Stigmatized Group Motivate One to Help the Group? Personality and Social Psychology Bulletin 28, 12 (2002), 1656-1666. https://doi.org/10.1177/014616702237647

[8] C Daniel Batson, Marina P Polycarpou, Eddie Harmon-Jones, Heidi J Imhoff, Erin C Mitchener, Lori L Bednar, Tricia R Klein, and Lori Highberger. 1997. Empathy and Attitudes: Can Feeling for a Member of a Stigmatized Group Improve Feelings Toward the Group? Journal of Personality and Social Psychology 72, 1 (1997), 105. https: //doi.org/10.1037/0022-3514.72.1.105

[9] Chris Bevan, David Philip Green, Harry Farmer, Mandy Rose, Kirsten Cater, Danaë Stanton Fraser, and Helen Brown. 2019. Behind the Curtain of the "Ultimate Empathy Machine": On the Composition of Virtual Reality Nonfiction Experiences. In Proceedings of the 2019 CHI Conference on Human Factors in Computing Systems. ACM, 506. https: //doi.org/10.1145/3290605.3300736

[10] Audrey Bryan, Rosalyn Moran, Eimear Farrell, and Mary O’Brien. 2000. Drug-Related Knowledge, Attitudes and Beliefs in Ireland: Report of a Nation wide Survey. Health Research Board (HRB).

[11] Moira Burke and Mike Develin. 2016. Once More with Feeling: Supportive Responses to Social Sharing on Facebook. In Proceedings of the 19th ACM Conference on Computer-Supported Cooperative Work \& Social Computing. ACM, 1462-1474. https://doi.org/10.1145/2818048.2835199

[12] C Daryl Cameron, Lasana T Harris, and B Keith Payne. 2016. The Emotional Cost of Humanity: Anticipated Exhaustion Motivates Dehumanization of Stigmatized Targets. Social Psychological and Personality Science 7, 2 (2016), 105-112. https://doi.org/10.1177/1948550615604453

[13] L Mark Carrier, Alexander Spradlin, John P Bunce, and Larry D Rosen. 2015. Virtual empathy: Positive and negative impacts of going online upon empathy in young adults. Computers in Human Behavior 52 (2015), 39-48. https: //doi.org/10.1016/j.chb.2015.05.026

[14] Mina Cikara, Emile Bruneau, Jay J Van Bavel, and Rebecca Saxe. 2014. Their pain gives us pleasure: How intergroup dynamics shape empathic failures and counter-empathic responses. Fournal of Experimental Social Psychology 55 (2014), 110-125. https://doi.org/10.1016/j.jesp.2014.06.007

[15] Mina Cikara, Emile G Bruneau, and Rebecca R Saxe. 2011. Us and Them: Intergroup Failures of Empathy. Current Directions in Psychological Science 20, 3 (2011), 149-153. https://doi.org/10.1177/0963721411408713

[16] Patrick W Corrigan, Sachiko A Kuwabara, and John O’Shaughnessy. 2009. The Public Stigma of Mental Illness and Drug Addiction: Findings from a Stratified Random Sample. Journal of Social Work 9, 2 (2009), 139-147. https:

Proc. ACM Hum.-Comput. Interact., Vol. 3, No. CSCW, Article 201. Publication date: November 2019. 
//doi.org/10.1177/1468017308101818

[17] John A Cunningham, Linda C Sobell, Mark B Sobell, Sangeeta Agrawal, and Tony Toneatto. 1993. Barriers to treatment: Why alcohol and drug abusers delay or never seek treatment. Addictive Behaviors 18, 3 (1993), $347-353$. https://doi.org/10.1016/0306-4603(93)90036-9

[18] Franco Curmi, Maria Angela Ferrario, Jen Southern, and Jon Whittle. 2013. HeartLink: open broadcast of live biometric data to social networks. In Proceedings of the SIGCHI Conference on Human Factors in Computing Systems. ACM, 1749-1758. https://doi.org/10.1145/2470654.2466231

[19] Max T. Curran, Jeremy Raboff Gordon, Lily Lin, Priyashri Kamlesh Sridhar, and John Chuang. 2019. Understanding Digitally-Mediated Empathy: An Exploration of Visual, Narrative, and Biosensory Informational Cues. In Proceedings of the 2019 ACM Conference on Human Factors in Computing Systems. ACM, 614:1-614:13. https://doi.org/10.1145/ 3290605.3300844

[20] Mark H Davis, Laura Conklin, Amy Smith, and Carol Luce. 1996. Effect of perspective taking on the cognitive representation of persons: A merging of self and other. Journal of Personality and Social Psychology 70, 4 (1996), 713 https://doi.org/10.1037/0022-3514.70.4.713

[21] Jean Decety, Stephanie Echols, and Joshua Correll. 2010. The Blame Game: The Effect of Responsibility and Social Stigma on Empathy for Pain. Journal of Cognitive Neuroscience 22, 5 (2010), 985-997. https://doi.org/10.1162/jocn.2009.21266

[22] Alan R Dennis and Susan T Kinney. 1998. Testing Media Richness Theory in the New Media: The Effects of Cues, Feedback, and Task Equivocality. Information Systems Research 9, 3 (1998), 256-274. https://doi.org/10.1287/isre.9.3.256

[23] Carroll Doherty, Jocelyn Kiley, and Bridget Johnson. 2016. Partisanship and Political Animosity in 2016. Pew Research Center (2016).

[24] Nancy Eisenberg and Paul A Miller. 1987. The Relation of Empathy to Prosocial and Related Behaviors. Psychological Bulletin 101, 1 (1987), 91. https://doi.org/10.1037/0033-2909.101.1.91

[25] FBI. 2017. 2017 Hate Crime Statistics. https://ucr.fbi.gov/hate-crime/2017

[26] Susan T Fiske. 2013. Interdependence and the reduction of prejudice. In Reducing prejudice and discrimination. Psychology Press, 125-146.

[27] Susan T Fiske, Amy JC Cuddy, Peter Glick, and Jun Xu. 2002. A model of (often mixed) stereotype content: competence and warmth respectively follow from perceived status and competition. Fournal of Personality and Social Psychology 82, 6 (2002), 878. https://doi.org/10.1037/0022-3514.82.6.878

[28] World Economic Forum. 2019. The Global Risks Report 2019 14th Edition. World Economic Forum. http://wef.ch/ risks2019

[29] Melike M Fourie, Sivenesi Subramoney, and Pumla Gobodo-Madikizela. 2017. A Less Attractive Feature of Empathy: Intergroup Empathy Bias. In Empathy-An Evidence-based Interdisciplinary Perspective. IntechOpen. https://doi.org/10 5772/intechopen.69287

[30] Jesse Fox, Carlos Cruz, and Ji Young Lee. 2015. Perpetuating online sexism offline: Anonymity, interactivity, and the effects of sexist hashtags on social media. Computers in Human Behavior 52 (2015), 436-442. https://doi.org/10.1016/j. chb.2015.06.024

[31] Thomas Fuchs. 2019. Empathy, Group Identity, and the Mechanisms of Exclusion: An Investigation into the Limits of Empathy. Topoi 38, 1 (2019), 239-250. https://doi.org/10.1007/s11245-017-9499-z

[32] Adam D Galinsky and Gordon B Moskowitz. 2000. Perspective-taking: Decreasing stereotype expression, stereotype accessibility, and in-group favoritism. Journal of Personality and Social Psychology 78, 4 (2000), 708. https://doi.org/10 1037//0022-3514.78.4.708

[33] Erving Goffman. 1963. Stigma: Notes on the Management of Spoiled Identity. Printice Hall.

[34] Charlotte N Gunawardena. 1995. Social Presence Theory and Implications for Interaction and Collaborative Learning in Computer Conferences. International fournal of Educational Telecommunications 1, 2 (1995), 147-166.

[35] Mariam Hassib, Daniel Buschek, Paweł W Wozniak, and Florian Alt. 2017. HeartChat: Heart Rate Augmented Mobile Chat to Support Empathy and Awareness. In Proceedings of the 2017 CHI Conference on Human Factors in Computing Systems. ACM, 2239-2251. https://doi.org/10.1145/3025453.3025758

[36] Fernanda Herrera, Jeremy Bailenson, Erika Weisz, Elise Ogle, and Jamil Zaki. 2018. Building long-term empathy: A large-scale comparison of traditional and virtual reality perspective-taking. PloS One 13, 10 (2018), e0204494. https://doi.org/10.1371/journal.pone.0204494

[37] Noura Howell, John Chuang, Abigail De Kosnik, Greg Niemeyer, and Kimiko Ryokai. 2018. Emotional Biosensing: Exploring Critical Alternatives. Proceedings of the ACM on Human-Computer Interaction 2, CSCW (2018), 69. https: //doi.org/10.1145/3274338

[38] Noura Howell, Laura Devendorf, Rundong Kevin Tian, Tomás Vega Galvez, Nan-Wei Gong, Ivan Poupyrev, Eric Paulos, and Kimiko Ryokai. 2016. Biosignals as Social Cues: Ambiguity and Emotional Interpretation in Social Displays of Skin Conductance. In Proceedings of the 2016 ACM Conference on Designing Interactive Systems. ACM, 865-870. 
[39] Noura Howell, Laura Devendorf, Tomás Alfonso Vega Gálvez, Rundong Tian, and Kimiko Ryokai. 2018. Tensions of Data-Driven Reflection: A Case Study of Real-Time Emotional Biosensing. In Proceedings of the 2018 CHI Conference on Human Factors in Computing Systems. ACM, 431. https://doi.org/10.1145/3173574.3174005

[40] Joris H Janssen, Jeremy N Bailenson, Wijnand A IJsselsteijn, and Joyce HDM Westerink. 2010. Intimate Heartbeats: Opportunities for Affective Communication Technology. IEEE Transactions on Affective Computing 2 (2010), 72-80. https://doi.org/10.1109/T-AFFC.2010.13

[41] Shagun Jhaver, Larry Chan, and Amy Bruckman. 2017. The View from the Other Side: The Border Between Controversial Speech and Harassment on Kotaku in Action. First Monday (2017). https://doi.org/10.5210/fm.v23i2.8232

[42] Edward Jones, Amerigo Farina, Albert Hastorf, Hazel Markus, Dale T. Miller, and Robert Scott. 1984. Social Stigma: The Psychology of Marked Relationships. Freeman and Company.

[43] Geoff F Kaufman and Lisa K Libby. 2012. Changing Beliefs and Behavior Through Experience-Taking. fournal of Personality and Social Psychology 103, 1 (2012), 1. https://doi.org/10.1037/a0027525

[44] Sara H Konrath, Edward H O’Brien, and Courtney Hsing. 2011. Changes in Dispositional Empathy in American College Students Over Time: A Meta-Analysis. Personality and Social Psychology Review 15, 2 (2011), 180-198. https://doi.org/10.1177/1088868310377395

[45] Martijn JL Kors, Gabriele Ferri, Erik D Van Der Spek, Cas Ketel, and Ben AM Schouten. 2016. A Breathtaking Journey. On the Design of an Empathy-Arousing Mixed-Reality Game. In Proceedings of the 2016 Annual Symposium on Computer-Human Interaction in Play. ACM, 91-104. https://doi.org/10.1145/2967934.2968110

[46] Karel Kreijns, Paul A Kirschner, Wim Jochems, and Hans Van Buuren. 2011. Measuring perceived social presence in distributed learning groups. Education and Information Technologies 16, 4 (2011), 365-381. https://doi.org/10.1007/ s10639-010-9135-7

[47] Youngho Lee, Katsutoshi Masai, Kai Kunze, Maki Sugimoto, and Mark Billinghurst. 2016. A Remote Collaboration System with Empathy Glasses. In 2016 IEEE International Symposium on Mixed and Augmented Reality (ISMAR-Adjunct). IEEE, 342-343. https://doi.org/10.1109/ISMAR-Adjunct.2016.0112

[48] Ruoyun Lin and Sonja Utz. 2017. Self-disclosure on SNS: Do disclosure intimacy and narrativity influence interpersonal closeness and social attraction? Computers in Human Behavior 70 (2017), 426-436. https://doi.org/10.1016/j.chb.2017. 01.012

[49] Fannie Liu, Laura Dabbish, and Geoff Kaufman. 2017. Can Biosignals be Expressive? How Visualizations Affect Impression Formation from Shared Brain Activity. Proceedings of the ACM on Human-Computer Interaction 1, CSCW (2017), 71:1-71:21. https://doi.org/10.1145/3134706

[50] Fannie Liu, Laura Dabbish, and Geoff Kaufman. 2017. Supporting Social Interactions with an Expressive Heart Rate Sharing Application. Proceedings of the ACM on Interactive, Mobile, Wearable and Ubiquitous Technologies 1, 3 (2017), 77:1-77:26. https://doi.org/10.1145/3130943

[51] Fannie Liu, Mario Esparza, Maria Pavlovskaia, Geoff Kaufman, Laura Dabbish, and Andrés Monroy-Hernández. 2019. Animo: Sharing Biosignals on a Smartwatch for Lightweight Social Connection. Proceedings of the ACM on Interactive, Mobile, Wearable and Ubiquitous Technologies 3, 1 (2019), 18:1-18:19. https://doi.org/10.1145/3314405

[52] Charlie Lloyd. 2013. The stigmatization of problem drug users: A narrative literature review. Drugs: Education, Prevention and Policy 20, 2 (2013), 85-95. https://doi.org/10.3109/09687637.2012.743506

[53] Nick Merrill and Coye Cheshire. 2016. Habits of the Heart (rate): Social Interpretation of Biosignals in Two Interaction Contexts. In Proceedings of the 19th International Conference on Supporting Group Work. ACM, 31-38. https://doi.org/ $10.1145 / 2957276.2957313$

[54] Nick Merrill and Coye Cheshire. 2017. Trust Your Heart: Assessing Cooperation and Trust with Biosignals in ComputerMediated Interactions. In Proceedings of the 2017 ACM Conference on Computer Supported Cooperative Work and Social Computing. ACM, 2-12. https://doi.org/10.1145/2998181.2998286

[55] Chris Milk. 2015. How virtual reality can create the ultimate empathy machine. https://www.ted.com/talks/chris milk_how_virtual_reality_can_create_the_ultimate_empathy_machine

[56] Hyeryung Christine Min and Tek-Jin Nam. 2014. Biosignal sharing for affective connectedness. In CHI'14 Extended Abstracts on Human Factors in Computing Systems. ACM, 2191-2196. https://doi.org/10.1145/2559206.2581345

[57] David T Nguyen and John Canny. 2009. More than face-to-face: Empathy effects of video framing. In Proceedings of the SIGCHI Conference on Human Factors in Computing Systems. ACM, 423-432. https://doi.org/10.1145/1518701.1518770

[58] Kristine L Nowak and Frank Biocca. 2003. The Effect of the Agency and Anthropomorphism on Users' Sense of Telepresence, Copresence, and Social Presence in Virtual Environments. Presence: Teleoperators \& Virtual Environments 12, 5 (2003), 481-494. https://doi.org/10.1162/105474603322761289

[59] Yoshimatsu Saito, Atsushi Ueshima, Shigehito Tanida, and Tatsuya Kameda. 2019. How does social information affect charitable giving?: Empathic concern promotes support for underdog recipient. Social Neuroscience (2019), 1-14. https://doi.org/10.1080/17470919.2019.1599421 
[60] Timothy J Saunders, Alex H Taylor, and Quentin D Atkinson. 2016. No evidence that a range of artificial monitoring cues influence online donations to charity in an MTurk sample. Royal Society Open Science 3, 10 (2016). https: //doi.org/10.1098/rsos.150710

[61] Holger Schnädelbach, Stefan Rennick Egglestone, Stuart Reeves, Steve Benford, Brendan Walker, and Michael Wright. 2008. Performing Thrill: Designing Telemetry Systems and Spectator Interfaces for Amusement Rides. In Proceedings of the SIGCHI Conference on Human Factors in Computing Systems. ACM, 1167-1176. https://doi.org/10.1145/1357054 1357238

[62] J. Short, E. Williams, and B. Christie. 1976. The Social Psychology of Telecommunications. Wiley. https://books.google. com/books?id=Ze63AAAAIAAJ

[63] Petr Slovák, Joris Janssen, and Geraldine Fitzpatrick. 2012. Understanding Heart Rate Sharing: Towards Unpacking Physiosocial Space. In Proceedings of the SIGCHI Conference on Human Factors in Computing Systems. ACM, 859-868. https://doi.org/10.1145/2207676.2208526

[64] Brandon Stanton. 2019. Humans of New York. https://www.humansofnewyork.com

[65] Haruto Takagishi, Michiko Koizumi, Takayuki Fujii, Joanna Schug, Shinya Kameshima, and Toshio Yamagishi. 2014. The Role of Cognitive and Emotional Perspective Taking in Economic Decision Making in the Ultimatum Game. PloS One 9, 9 (2014), e108462. https://doi.org/10.1371/journal.pone.0108462

[66] Laura E Thomas and Daniel Pemstein. 2015. What You See is What You Get: Webcam Placement Influences Perception and Social Coordination. Frontiers in Psychology 6 (2015), 306. https://doi.org/10.3389/fpsyg.2015.00306

[67] Leonieke C Van Boekel, Evelien PM Brouwers, Jaap Van Weeghel, and Henk FL Garretsen. 2013. Stigma among health professionals towards patients with substance use disorders and its consequences for healthcare delivery: systematic review. Drug and Alcohol Dependence 131, 1-2 (2013), 23-35. https://doi.org/10.1016/j.drugalcdep.2013.02.018

[68] David Watson, Lee Anna Clark, and Auke Tellegen. 1988. Development and validation of brief measures of positive and negative affect: the PANAS scales. Fournal of Personality and Social Psychology 54, 6 (1988), 1063.

[69] Julia Werner, Reto Wettach, and Eva Hornecker. 2008. United-pulse: Feeling your partner's pulse. In Proceedings of the 10th Conference on Human-Computer Interaction with Mobile Devices and Services. ACM, 535-538. https: //doi.org/10.1145/1409240.1409338

[70] S Christian Wheeler, W Blair G Jarvis, and Richard E Petty. 2001. Think Unto Others: The Self-Destructive Impact of Negative Racial Stereotypes. Journal of Experimental Social Psychology 37, 2 (2001), 173-180. https://doi.org/10.1006/ jesp.2000.1448

[71] Richmond Y Wong, Deirdre K Mulligan, Ellen Van Wyk, James Pierce, and John Chuang. 2017. Eliciting Values Reflections by Engaging Privacy Futures Using Design Workbooks. Proceedings of the ACM on Human-Computer Interaction 1, CSCW (2017), 111. https://doi.org/10.1145/3134746

[72] Lawrence H Yang, Liang Y Wong, Margaux M Grivel, and Deborah S Hasin. 2017. Stigma and substance use disorders: an international phenomenon. Current Opinion in Psychiatry 30, 5 (2017), 378-388. https://doi.org/10.1097/YCO. 0000000000000351

[73] Jamil Zaki and Mina Cikara. 2015. Addressing Empathic Failures. Current Directions in Psychological Science 24, 6 (2015), 471-476. https://doi.org/10.1177/0963721415599978

Received April 2019; revised June 2019; accepted August 2019 